International Journal of Modern Physics E (C) World Scientific Publishing Company

\title{
$\beta$-decay of key titanium isotopes in stellar environment
}

\author{
Jameel-Un Nabi* \\ Faculty of Engineering Sciences, GIK Institute of Engineering Sciences and Technology, Topi \\ 23640, Khyber Pakhtunkhwa, Pakistan \\ jameel@giki.edu.pk \\ Irgaziev Bakhadir \\ Faculty of Engineering Sciences, GIK Institute of Engineering Sciences and Technology, Topi \\ 23640, Khyber Pakhtunkhwa, Pakistan \\ bakhadir@giki.edu.pk \\ Received (received date) \\ Revised (revised date)
}

\begin{abstract}
Amongst iron regime nuclei, $\beta$-decay rates on titanium isotopes are considered to be important during the late phases of evolution of massive stars. The key $\beta$-decay isotopes during presupernova evolution were searched from available literature and a microscopic calculation of the decay rates were performed using the proton-neutron quasiparticle random phase approximation (pn-QRPA) theory. As per earlier simulation results electron capture and $\beta$-decay on certain isotopes of titanium are considered to be important for the presupernova evolution of massive stars. Earlier the stellar electron capture rates and neutrino energy loss rates due to relevant titanium isotopes were presented. In this paper we finally present the $\beta$-decay rates of key titanium isotopes in stellar environment. The results are also compared against previous calculations. The pn-QRPA $\beta$-decay rates are bigger at high stellar temperatures and smaller at high stellar densities compared to the large scale shell model results. This study can prove useful for the core-collapse simulators.
\end{abstract}

PACS Number(s): 97.10.Cv, 26.50.+x, 23.40.-s, 23.40.Bw, 21.60.Jz

\section{Introduction}

Shortly after the discovery of the Gamow-Teller (GT) resonance, Bethe and collaborators 1 suggested its importance for stellar weak-interaction mediated reactions. The calculations of stellar $\beta$-decays and electron captures are very sensitive to the distribution and the total strength of the GT giant resonance. The centroid of the GT distribution functions determines the effective energy of the capture and decay reactions. Due to these weak interaction processes the value of $Y_{e}$ (lepton-to-baryon ratio) for a massive star changes from 1 (during hydrogen burning) to roughly 0.5

${ }^{*}$ Corresponding author. 
(at the beginning of carbon burning) and finally to around 0.42 just before the collapse to a supernova explosion. $\beta$-decay are relatively unimportant at the beginning of carbon burning phase of massive stars $\left(Y_{e} \sim 0.5\right)$. However it becomes increasingly competitive for neutron-rich nuclei due to an increase in phase space related to larger $\mathrm{Q}_{\beta}$ values. In earlier simulations of core-collapse studies $\beta$-decay of heavy nuclei were not considered. However it was soon acknowledged that during the early stages of the collapse, a strong $\beta$-decay rate can contribute both to the cooling rate (via the stellar antineutrinos produced) and to a much larger value of $Y_{e}$ at the formation of homologous core. This in turn would result in a smaller envelope and to a more energetic shock wave. Aufderheide and collaborators ${ }^{[2}$ stressed on the importance of $\beta$-decay rates in the iron core prior to the collapse. The authors also found in their calculation that the $\beta$-decay rates were strong enough and at times larger than the competing electron capture rates for $Y_{e} \sim 0.42-0.46$. The temporal variation of $Y_{e}$ within the core of a massive star has a pivotal role to play in the stellar evolution and a fine-tuning of this parameter at various stages of presupernova evolution is the key to generate an explosion. Collapse simulators world-wide find it challenging to transform the collapse of the core of massive stars to an explosion and to date there have been no successfully simulated spherically symmetric explosions. Even the 2D simulations (addition of convection) performed with a Boltzmann solver for the neutrino transport fails to convert the collapse into an explosion 3. (It is worth mentioning that recently a few simulation groups (e.g. Refs. 4/516) have reported successful explosions in 2D mode.) Additional energy sources (e.g. magnetic fields and rotations) were also sought that might transport energy to the mantle and lead to an explosion. World-wide core-collapse simulators are still working hard to come up with a convincing and decisive mode of producing explosions.

The calculation of stellar weak interaction rates has been performed by a number of authors during 1960's and 1970's. The rates were calculated using the best available physics of the time. However the first breakthrough was achieved in 1980 when Fuller, Fowler and Newman (commonly referred to as FFN) ${ }^{7}$ used a simple shell model to compute the location and strength of the GT resonances for 226 nuclei in the mass range $21 \leq A \leq 60$. FFN estimated the GT contributions to the rates by a parametrization based on the independent particle model. Aufderheide et al. 2 later extended the FFN work for heavier nuclei with $\mathrm{A}>60$ and took into consideration the quenching of the GT strength neglected by FFN.

Experimentally it has been shown that for $(p, n)$ and $(n, p)$ reactions the $0^{0}$ cross sections for such transitions are proportional to the squares of the matrix elements for the GT $\beta$ decay between the same states (e.g. Ref. 8 ). Results of such measurements disclosed that, in contrast to the independent particle model, the total GT strength is quenched and fragmented over many final states in the daughter nucleus caused by the residual nucleon-nucleon correlations. Both these effects are caused by the residual interaction among the valence nucleons and an 
accurate description of these correlations is essential for a reliable evaluation of the stellar weak interaction rates due to the strong phase space energy dependence. The measured data from various $(p, n)$ and $(n, p)$ experiments also revealed the misplacement of the GT centroid adopted in the parameterizations of FFN and subsequently used in the calculation of weak rates by Ref. 2 . Since then theoretical efforts were concentrated on the microscopic calculations of weak-interaction mediated rates of iron-regime nuclide. Two such widely used models are the largescale shell model (LSSM)(e.g. Ref. 9) ) and the proton-neutron quasiparticle random phase approximation (pn-QRPA) theory (e.g. Ref. 10). Both LSSM and pn-QRPA model later validated the finding of Ref. 2 , on microscopic grounds rather than phenomenological parametrization of GT centroids and strengths, that $\beta$-decay rates are larger than electron capture rates for a certain range of $Y_{e}$ values. The LSSM calculation, however, makes use of the so-called Brink's hypothesis in the electron capture direction and back-resonances in the $\beta$-decay direction to approximate the contributions from high-lying excited state GT strength distributions. Brink's hypothesis states that GT strength distribution on excited states is identical to that from ground state, shifted only by the excitation energy of the state. GT back resonances are the states reached by the strong GT transitions in the inverse process (electron capture) built on ground and excited states. $\beta$-decay rate calculation for iron-regime nuclei are also important during the late stages of stellar evolution.

Isotopes of titanium are amongst the key iron-regime nuclei that play a key role in the developments during the late phases of stellar evolution. A search was performed by authors of Ref. 2 for most important electron capture and $\beta$-decay nuclei after core silicon burning in massive stars. The authors assumed that the temperatures within the iron core were high enough for the strong and electromagnetic reactions to be in equilibrium. The nuclear Saha equation was then used to compute the isotopic abundances. They tabulated the 71 top $\beta$-decay nuclei averaged throughout the stellar trajectory for $0.40 \leq Y_{e} \leq 0.5$ (see Table 26 therein). From this table, six isotopes of titanium, namely ${ }^{51,52,53,54,55,56} \mathrm{Ti}$, were short-listed as those whose $\beta$-decay rates were suggested to have a significant contribution during and after core silicon burning phases in massive stars. A microscopic calculation of stellar $\beta$-decay rates was performed for these titanium isotopes using the pn-QRPA theory. Because of the high temperatures prevailing during the presupernova and supernova phase of a massive star, there is a reasonable probability of occupation of parent excited states and the total weak interaction rates have a finite contribution form these excited states. The pn-QRPA theory allows a microscopic state-by-state calculation of all these partial rates and this feature of the model greatly enhances the reliability of the calculated rates in stellar matter. The pn-QRPA model can handle any arbitrarily heavy system of nucleons as it has access to a luxurious model space of up to $7 \hbar \omega$ shells. The pn-QRPA model was successfully used to calculate weak interaction rates on important iron-regime nuclei (e.g. Refs. 10|11|12[13|14|15). Earlier Nabi and collaborators 16 presented a detailed analysis of the calculation of stellar electron capture rates on twenty two titanium isotopes. Later a calculation 
of neutrino and antineutrino energy loss rates in massive stars due to isotopes of titanium was also presented 17. In this paper we present the $\beta$-decay rates due to the above mentioned six isotopes of titanium in stellar matter. This completes the analysis of weak interaction rates of key titanium isotopes considered to be important for the presupernova evolution of massive stars. These microscopic rates can be of great utility for core-collapse simulators. The next section discusses briefly the formalism and presents the calculated $\beta$-decay rates. Comparison with previous calculations is also presented in this section. Section 3 finally summarizes the main conclusions.

\section{Calculations and Results}

The Hamiltonian of the pn-QRPA model and its diagonalization were discussed earlier in Ref. 16. The $\beta$-decay rates of a transition from the $i^{\text {th }}$ state of the parent to the $j^{\text {th }}$ state of the daughter nucleus are given by

$$
\lambda_{i j}^{\beta}=\left[\frac{\ln 2}{D}\right]\left[B(F)_{i j}+\left(g_{A} / g_{V}\right)^{2} B(G T)_{i j}\right]\left[f_{i j}^{\beta}\left(T, \rho, E_{f}\right)\right] .
$$

The value of $\mathrm{D}$ was taken to be $6295 \mathrm{~s} \frac{18}{\mathrm{~B}} \mathrm{~B}(\mathrm{~F})$ and $\mathrm{B}(\mathrm{GT})$ are reduced transition probabilities of the Fermi and Gamow-Teller (GT) transitions, respectively,

$$
\begin{gathered}
B(F)_{i j}=\frac{1}{2 J_{i}+1}\left|<j\left\|\sum_{k} t_{ \pm}^{k}\right\| i>\right|^{2} . \\
B(G T)_{i j}=\frac{1}{2 J_{i}+1}\left|<j\left\|\sum_{k} t_{ \pm}^{k} \vec{\sigma}^{k}\right\| i>\right|^{2} .
\end{gathered}
$$

Here $\vec{\sigma}^{k}$ is the spin operator and $t_{ \pm}^{k}$ stands for the isospin raising and lowering operator.

The $f_{i j}^{\beta}$ are the phase space integrals and are functions of stellar temperature $(T)$, density $(\rho)$ and Fermi energy $\left(E_{f}\right)$ of the electrons. They are explicitly given by

$$
f_{i j}^{\beta}=\int_{1}^{w_{m}} w \sqrt{w^{2}-1}\left(w_{m}-w\right)^{3} F(+Z, w)\left(1-G_{-}\right) d w,
$$

In Eq. (44), $w$ is the total energy of the electron including its rest mass. $w_{m}$ is the total $\beta$-decay energy,

$$
w_{m}=m_{p}-m_{d}+E_{i}-E_{j},
$$

where $m_{p}$ and $E_{i}$ are mass and excitation energies of the parent nucleus, and $m_{d}$ and $E_{j}$ of the daughter nucleus, respectively. $F(+Z, w)$ are the Fermi functions and were calculated according to the procedure adopted by Gove and Martin 19 . $G_{-}$ is the Fermi-Dirac distribution function for electrons. Details of the calculation of reduced transition probabilities can be found in Ref. 10. Construction of parent 
and daughter excited states and calculation of transition amplitudes between these states can be seen in Ref. 20 .

The total $\beta$-decay rate per unit time per nucleus is finally given by

$$
\lambda^{\beta}=\sum_{i j} P_{i} \lambda_{i j}^{\beta},
$$

where $P_{i}$ is the probability of occupation of parent excited states and follows the normal Boltzmann distribution. After the calculation of all partial rates for the transition $i \rightarrow j$ the summation was carried out over all initial and final states until satisfactory convergence was achieved in the rate calculation. The pn-QRPA theory allows a microscopic state-by-state calculation of both sums present in Eq. (6). This feature of the pn-QRPA model greatly increases the reliability of the calculated rates over other models in stellar matter where there exists a finite probability of occupation of excited states.

Stellar $\beta$-decay rates are sensitive functions of the available phase space, $\left(Q_{\beta}+\right.$ $\left.E_{i}-E_{j}\right)$. It is very much possible for nuclei with negative $Q_{\beta}$ values to undergo $\beta$ decays in stellar environment (strictly forbidden under terrestrial conditions) as the phase space can become positive depending on the calculated energy eigenvalues of the underlying theoretical model. The calculated $\beta$-decay rates of ${ }^{51,52,53,54,55,56} \mathrm{Ti}$ in stellar environment are presented in Table 1 . The calculated rates are tabulated on an abbreviated density scale. The first column gives $\log \left(\rho Y_{e}\right)$ in units of $\mathrm{gcm}^{-3}$, where $\rho$ is the baryon density and $Y_{e}$ is the ratio of the electron number to the baryon number. Stellar temperatures $\left(T_{9}\right)$ are stated in $10^{9} \mathrm{~K}$. The third column shows the calculated values of the Fermi energy in units of $M e V$. The last six columns give the calculated $\beta$-decay rates of selected titanium isotopes in units of $s^{-1}$. The calculated $\beta$-decay rates are tabulated in logarithmic (to base 10) scale. In the table, -100 means that the rate is smaller than $10^{-100} s^{-1}$. It can be seen from the table that at low stellar densities and temperatures, ${ }^{56} \mathrm{Ti}$ has the strongest $\beta$-decay rate whereas ${ }^{52} \mathrm{Ti}$ the weakest. As the core stiffens from density $\rho Y_{e}\left[\mathrm{gcm}^{-3}\right]=10$ to $10^{4}$ the $\beta$-decay rates does not change appreciably for a particular core temperature. As the core stiffens further the $\beta$-decay rates start decreasing by orders of magnitude because of the considerable reduction in available phase space. The $\beta$-decay rates increase monotonically with increasing temperature. Positron capture rates act in the same direction as $\beta$-decay rates and at times tend to compete with the later. The positron capture rates on these titanium isotopes were also calculated. Table 2 shows the ratio of the calculated positron capture to $\beta$-decay rates for isotopes of titanium at selected temperature and density points. It can be seen from this table that only at high stellar temperatures, $\mathrm{T}_{9}[K] \sim 30$, does the positron capture rate compete with the $\beta$-decay rates. For all other physical conditions the positron capture rates are smaller by many orders of magnitude and hence can be safely neglected as compared to the $\beta$-decay rates during the presupernova evolution of massive stars where the core temperature is considerably less. The complete electronic version (ASCII files) of these rates may be requested 
Table 1. $\beta$-decay rates of $51,52,53,54,55,56 \mathrm{Ti}$ for selected densities and temperatures in stellar matter. $\log \rho Y_{e}$ has units of $\mathrm{g} / \mathrm{cm}^{3}$, where $\rho$ is the baryon density and $Y_{e}$ is the ratio of the lepton number to the baryon number. Temperatures $\left(T_{9}\right)$ are given in units of $10^{9} \mathrm{~K}$. The calculated Fermi energy is denoted by $E_{f}$ and is given in units of $\mathrm{MeV}$. All calculated $\beta$-decay rates are tabulated in logarithmic (to base 10) scale in units of $s^{-1}$. In the table, -100 means that the rate is smaller than $10^{-100} \mathrm{~s}^{-1}$.

\begin{tabular}{|c|c|c|c|c|c|c|c|c|}
\hline $\log \rho Y_{e}$ & $T_{9}$ & $E_{f}$ & ${ }^{51} \mathrm{Ti}$ & ${ }^{52} \mathrm{Ti}$ & ${ }^{53} \mathrm{Ti}$ & ${ }^{54} \mathrm{Ti}$ & ${ }^{55} \mathrm{Ti}$ & ${ }^{56} \mathrm{Ti}$ \\
\hline 1.0 & 0.01 & 0.508 & -2.698 & -3.465 & -2.137 & -0.466 & -0.146 & 1.004 \\
\hline 1.0 & 0.10 & 0.453 & -2.732 & -3.465 & -2.137 & -0.466 & -0.146 & 1.004 \\
\hline 1.0 & 0.20 & 0.377 & -2.777 & -3.465 & -2.135 & -0.466 & -0.146 & 1.004 \\
\hline 1.0 & 0.40 & 0.205 & -2.812 & -3.465 & -2.106 & -0.466 & -0.145 & 1.004 \\
\hline 1.0 & 0.70 & 0.008 & -2.830 & -3.465 & -2.049 & -0.466 & -0.108 & 1.004 \\
\hline 1.0 & 1.00 & 0.000 & -2.834 & -3.465 & -2.012 & -0.466 & -0.009 & 1.004 \\
\hline 1.0 & 1.50 & 0.000 & -2.807 & -3.466 & -1.979 & -0.466 & 0.169 & 1.004 \\
\hline 1.0 & 2.00 & 0.000 & -2.739 & -3.468 & -1.958 & -0.466 & 0.292 & 1.004 \\
\hline 1.0 & 3.00 & 0.000 & -2.557 & -3.482 & -1.798 & -0.468 & 0.437 & 1.003 \\
\hline 1.0 & 5.00 & 0.000 & -2.074 & -3.220 & -0.913 & -0.471 & 0.594 & 1.002 \\
\hline 1.0 & 10.00 & 0.000 & -1.031 & -0.946 & 0.126 & 0.139 & 0.814 & 1.269 \\
\hline 1.0 & 30.00 & 0.000 & 0.221 & 0.699 & 1.172 & 1.424 & 1.616 & 2.138 \\
\hline 4.0 & 0.01 & 0.523 & -2.699 & -3.468 & -2.139 & -0.466 & -0.146 & 1.004 \\
\hline 4.0 & 0.10 & 0.516 & -2.733 & -3.467 & -2.138 & -0.466 & -0.146 & 1.004 \\
\hline 4.0 & 0.20 & 0.498 & -2.778 & -3.466 & -2.136 & -0.466 & -0.146 & 1.004 \\
\hline 4.0 & 0.40 & 0.444 & -2.813 & -3.466 & -2.107 & -0.466 & -0.145 & 1.004 \\
\hline 4.0 & 0.70 & 0.337 & -2.830 & -3.466 & -2.050 & -0.466 & -0.108 & 1.004 \\
\hline 4.0 & 1.00 & 0.209 & -2.834 & -3.466 & -2.013 & -0.466 & -0.009 & 1.004 \\
\hline 4.0 & 1.50 & 0.047 & -2.807 & -3.466 & -1.979 & -0.466 & 0.169 & 1.004 \\
\hline 4.0 & 2.00 & 0.014 & -2.739 & -3.468 & -1.958 & -0.466 & 0.292 & 1.004 \\
\hline 4.0 & 3.00 & 0.004 & -2.557 & -3.482 & -1.798 & -0.469 & 0.437 & 1.003 \\
\hline 4.0 & 5.00 & 0.001 & -2.074 & -3.220 & -0.913 & -0.471 & 0.594 & 1.002 \\
\hline 4.0 & 10.00 & 0.000 & -1.031 & -0.946 & 0.126 & 0.139 & 0.814 & 1.269 \\
\hline 4.0 & 30.00 & 0.000 & 0.221 & 0.699 & 1.172 & 1.424 & 1.616 & 2.138 \\
\hline 7.0 & 0.01 & 1.223 & -2.918 & -3.786 & -2.285 & -0.543 & -0.192 & 0.978 \\
\hline 7.0 & 0.10 & 1.222 & -2.946 & -3.786 & -2.285 & -0.543 & -0.192 & 0.979 \\
\hline 7.0 & 0.20 & 1.222 & -2.986 & -3.785 & -2.283 & -0.543 & -0.192 & 0.979 \\
\hline 7.0 & 0.40 & 1.219 & -3.015 & -3.783 & -2.242 & -0.543 & -0.191 & 0.979 \\
\hline 7.0 & 0.70 & 1.212 & -3.027 & -3.777 & -2.165 & -0.542 & -0.151 & 0.979 \\
\hline 7.0 & 1.00 & 1.200 & -3.024 & -3.768 & -2.116 & -0.541 & -0.044 & 0.979 \\
\hline 7.0 & 1.50 & 1.173 & -2.980 & -3.749 & -2.069 & -0.538 & 0.143 & 0.980 \\
\hline 7.0 & 2.00 & 1.133 & -2.886 & -3.726 & -2.037 & -0.535 & 0.272 & 0.981 \\
\hline 7.0 & 3.00 & 1.021 & -2.658 & -3.681 & -1.845 & -0.528 & 0.422 & 0.983 \\
\hline 7.0 & 5.00 & 0.698 & -2.109 & -3.275 & -0.924 & -0.507 & 0.585 & 0.989 \\
\hline 7.0 & 10.00 & 0.196 & -1.037 & -0.950 & 0.122 & 0.134 & 0.811 & 1.266 \\
\hline 7.0 & 30.00 & 0.021 & 0.221 & 0.699 & 1.171 & 1.423 & 1.615 & 2.138 \\
\hline 10.0 & 0.01 & 11.118 & -100 & -100 & -100 & -100 & -100 & -100 \\
\hline 10.0 & 0.10 & 11.118 & -100 & -100 & -100 & -100 & -100 & -100 \\
\hline 10.0 & 0.20 & 11.118 & -100 & -100 & -100 & -100 & -87.308 & -93.598 \\
\hline 10.0 & 0.40 & 11.118 & -100 & -100 & -74.497 & -85.977 & -45.263 & -48.401 \\
\hline 10.0 & 0.70 & 11.117 & -62.404 & -65.400 & -43.640 & -50.168 & -26.906 & -28.699 \\
\hline 10.0 & 1.00 & 11.116 & -44.379 & -46.217 & -31.133 & -35.642 & -19.400 & -20.626 \\
\hline 10.0 & 1.50 & 11.113 & -30.186 & -31.101 & -21.236 & -24.133 & -13.413 & -14.125 \\
\hline 10.0 & 2.00 & 11.110 & -22.978 & -23.418 & -16.171 & -18.229 & -10.322 & -10.715 \\
\hline 10.0 & 3.00 & 11.099 & -15.627 & -15.575 & -10.948 & -12.114 & -7.096 & -7.084 \\
\hline 10.0 & 5.00 & 11.063 & -9.553 & -9.075 & -6.539 & -6.902 & -4.308 & -3.841 \\
\hline 10.0 & 10.00 & 10.898 & -4.718 & -3.878 & -2.894 & -2.530 & -1.871 & -0.907 \\
\hline 10.0 & 30.00 & 9.163 & -0.701 & -0.090 & 0.362 & 0.693 & 0.866 & 1.471 \\
\hline 11.0 & 0.01 & 23.934 & -100 & -100 & -100 & -100 & -100 & -100 \\
\hline 11.0 & 0.10 & 23.934 & -100 & -100 & -100 & -100 & -100 & -100 \\
\hline 11.0 & 0.20 & 23.934 & -100 & -100 & -100 & -100 & -100 & -100 \\
\hline 11.0 & 0.40 & 23.934 & -100 & -100 & -100 & -100 & -100 & -100 \\
\hline 11.0 & 0.70 & 23.934 & -100 & -100 & -100 & -100 & -100 & -100 \\
\hline 11.0 & 1.00 & 23.933 & -100 & -100 & -95.730 & -100 & -83.987 & -85.027 \\
\hline 11.0 & 1.50 & 23.932 & -73.255 & -74.170 & -64.305 & -67.176 & -56.474 & -57.036 \\
\hline 11.0 & 2.00 & 23.930 & -55.285 & -55.725 & -48.478 & -50.515 & -42.622 & -42.893 \\
\hline 11.0 & 3.00 & 23.925 & -37.175 & -37.122 & -32.496 & -33.647 & -28.639 & -28.536 \\
\hline 11.0 & 5.00 & 23.908 & -22.501 & -22.023 & -19.487 & -19.840 & -17.252 & -16.709 \\
\hline 11.0 & 10.00 & 23.832 & -11.236 & -10.393 & -9.411 & -9.033 & -8.380 & -7.344 \\
\hline 11.0 & 30.00 & 23.016 & -2.980 & -2.334 & -1.889 & -1.527 & -1.361 & -0.703 \\
\hline
\end{tabular}


Table 2. Ratio of calculated positron capture rates to $\beta$-decay rates for $51,52,53,54,55,56 \mathrm{Ti}$ for selected densities and temperatures in stellar matter. $\log \rho Y_{e}$ has units of $\mathrm{g} / \mathrm{cm}^{3}$, where $\rho$ is the baryon density and $Y_{e}$ is the ratio of the lepton number to the baryon number. Temperatures $\left(T_{9}\right)$ are given in units of $10^{9} \mathrm{~K}$. The calculated Fermi energy is denoted by $E_{f}$ and is given in units of $\mathrm{MeV}$. Cases with no reported ratio imply that either (or both) of the calculated rate(s) is (are) smaller than $10^{-100} s^{-1}$.

\begin{tabular}{|ccc|cccccc|}
$\log \rho Y_{e}$ & $T_{9}$ & $E_{f}$ & ${ }^{51} \mathrm{Ti}$ & ${ }^{52} \mathrm{Ti}$ & ${ }^{53} \mathrm{Ti}$ & ${ }^{54} \mathrm{Ti}$ & ${ }^{55} \mathrm{Ti}$ & ${ }^{56} \mathrm{Ti}$ \\
\hline 1.0 & 0.10 & 0.453 & $1.20 \mathrm{E}-53$ & $5.42 \mathrm{E}-52$ & $3.18 \mathrm{E}-53$ & $5.82 \mathrm{E}-54$ & $2.89 \mathrm{E}-54$ & $1.04 \mathrm{E}-54$ \\
1.0 & 1.00 & 0.000 & $2.70 \mathrm{E}-05$ & $1.78 \mathrm{E}-03$ & $7.21 \mathrm{E}-05$ & $1.50 \mathrm{E}-05$ & $5.52 \mathrm{E}-06$ & $2.51 \mathrm{E}-06$ \\
1.0 & 3.00 & 0.000 & $1.46 \mathrm{E}-02$ & $2.32 \mathrm{E}+00$ & $5.20 \mathrm{E}-02$ & $1.23 \mathrm{E}-02$ & $1.79 \mathrm{E}-03$ & $1.75 \mathrm{E}-03$ \\
1.0 & 10.00 & 0.000 & $1.25 \mathrm{E}+00$ & $2.49 \mathrm{E}+00$ & $4.20 \mathrm{E}-01$ & $7.85 \mathrm{E}-01$ & $1.94 \mathrm{E}-01$ & $1.69 \mathrm{E}-01$ \\
1.0 & 30.00 & 0.000 & $2.25 \mathrm{E}+02$ & $5.55 \mathrm{E}+01$ & $6.01 \mathrm{E}+01$ & $3.07 \mathrm{E}+01$ & $3.02 \mathrm{E}+01$ & $1.15 \mathrm{E}+01$ \\
4.0 & 0.10 & 0.516 & $8.04 \mathrm{E}-57$ & $3.64 \mathrm{E}-55$ & $2.13 \mathrm{E}-56$ & $3.90 \mathrm{E}-57$ & $1.93 \mathrm{E}-57$ & $6.98 \mathrm{E}-58$ \\
4.0 & 1.00 & 0.209 & $2.41 \mathrm{E}-06$ & $1.59 \mathrm{E}-04$ & $6.44 \mathrm{E}-06$ & $1.34 \mathrm{E}-06$ & $4.92 \mathrm{E}-07$ & $2.24 \mathrm{E}-07$ \\
4.0 & 3.00 & 0.004 & $1.44 \mathrm{E}-02$ & $2.29 \mathrm{E}+00$ & $5.13 \mathrm{E}-02$ & $1.21 \mathrm{E}-02$ & $1.76 \mathrm{E}-03$ & $1.73 \mathrm{E}-03$ \\
4.0 & 10.00 & 0.000 & $1.25 \mathrm{E}+00$ & $2.49 \mathrm{E}+00$ & $4.20 \mathrm{E}-01$ & $7.85 \mathrm{E}-01$ & $1.94 \mathrm{E}-01$ & $1.69 \mathrm{E}-01$ \\
4.0 & 30.00 & 0.000 & $2.25 \mathrm{E}+02$ & $5.55 \mathrm{E}+01$ & $6.01 \mathrm{E}+01$ & $3.08 \mathrm{E}+01$ & $3.02 \mathrm{E}+01$ & $1.15 \mathrm{E}+01$ \\
7.0 & 0.10 & 1.222 & $3.37 \mathrm{E}-92$ & $1.95 \mathrm{E}-90$ & $7.67 \mathrm{E}-92$ & $1.19 \mathrm{E}-92$ & $5.51 \mathrm{E}-93$ & $1.90 \mathrm{E}-93$ \\
7.0 & 1.00 & 1.200 & $3.75 \mathrm{E}-11$ & $3.21 \mathrm{E}-09$ & $8.20 \mathrm{E}-11$ & $1.60 \mathrm{E}-11$ & $5.37 \mathrm{E}-12$ & $2.38 \mathrm{E}-12$ \\
7.0 & 3.00 & 1.021 & $3.62 \mathrm{E}-04$ & $7.18 \mathrm{E}-02$ & $1.13 \mathrm{E}-03$ & $2.77 \mathrm{E}-04$ & $3.64 \mathrm{E}-05$ & $3.61 \mathrm{E}-05$ \\
7.0 & 10.00 & 0.196 & $1.01 \mathrm{E}+00$ & $2.02 \mathrm{E}+00$ & $3.40 \mathrm{E}-01$ & $6.37 \mathrm{E}-01$ & $1.57 \mathrm{E}-01$ & $1.37 \mathrm{E}-01$ \\
7.0 & 30.00 & 0.021 & $2.24 \mathrm{E}+02$ & $5.51 \mathrm{E}+01$ & $5.98 \mathrm{E}+01$ & $3.05 \mathrm{E}+01$ & $3.01 \mathrm{E}+01$ & $1.14 \mathrm{E}+01$ \\
11.0 & 0.10 & 23.934 & - & - & - & - & - & - \\
11.0 & 1.00 & 23.933 & - & - & - & - & - & - \\
11.0 & 3.00 & 23.925 & $3.97 \mathrm{E}-08$ & $6.61 \mathrm{E}-07$ & $1.69 \mathrm{E}-11$ & $1.21 \mathrm{E}-09$ & $1.40 \mathrm{E}-14$ & $3.97 \mathrm{E}-14$ \\
11.0 & 10.00 & 23.832 & $1.99 \mathrm{E}-02$ & $7.00 \mathrm{E}-03$ & $1.46 \mathrm{E}-03$ & $1.18 \mathrm{E}-03$ & $3.10 \mathrm{E}-04$ & $7.13 \mathrm{E}-05$ \\
11.0 & 30.00 & 23.016 & $4.97 \mathrm{E}+01$ & $8.36 \mathrm{E}+00$ & $9.66 \mathrm{E}+00$ & $3.85 \mathrm{E}+00$ & $4.02 \mathrm{E}+00$ & $1.13 \mathrm{E}+01$
\end{tabular}

from the corresponding author.

The calculation of $\beta$-decay rates was also compared with previous calculations. For the sake of comparison we considered the pioneer calculations of FFN ${ }^{7}$ and those performed using the large-scale shell model (LSSM) 9]. Figure 1 depicts the comparison of $\beta$-decay rates of ${ }^{51} \mathrm{Ti}$ with earlier calculations. The upper panel displays the ratio of calculated rates to the LSSM rates, $R_{\beta}(Q R P A / L S S M)$, while the lower panel shows the corresponding comparison between FFN and LSSM calculations, $R_{\beta}(F F N / L S S M)$. All graphs are drawn at four selected values of stellar densities $\left(\rho Y_{e}\left[\mathrm{gcm}^{-3}\right]=10^{1}, 10^{4}, 10^{7}\right.$ and $\left.10^{11}\right)$. These values correspond roughly to low, medium-low, medium-high and high stellar densities, respectively. The selected values for temperature on the abscissa are $\mathrm{T}_{9}[K]=1,1.5,2,3,5,10$ and 30 . It can be seen from Figure 1 that the pn-QRPA calculated $\beta$-decay rates of ${ }^{51} \mathrm{Ti}$ are generally in good agreement with LSSM rates (within a factor 5). The comparison between FFN and LSSM calculations is also good except at high temperatures and densities. FFN did not take into effect the process of particle emission from excited states and their parent excitation energies extended well beyond the particle decay channel. The occupation probability of these high lying excited states becomes finite at high temperatures and densities. FFN rates are consequently bigger by more than one order of magnitude at $\mathrm{T}_{9}[K]=30$.

For the case of $\beta$-decay rates of ${ }^{52} \mathrm{Ti}$, the LSSM rates are slightly bigger (Figure 2) compared to the reported decay rates. At low densities the LSSM decay rates are bigger by up to a factor of five whereas at high densities they are bigger by as much as factor of twenty. Whereas the individual discrete transitions between ini- 


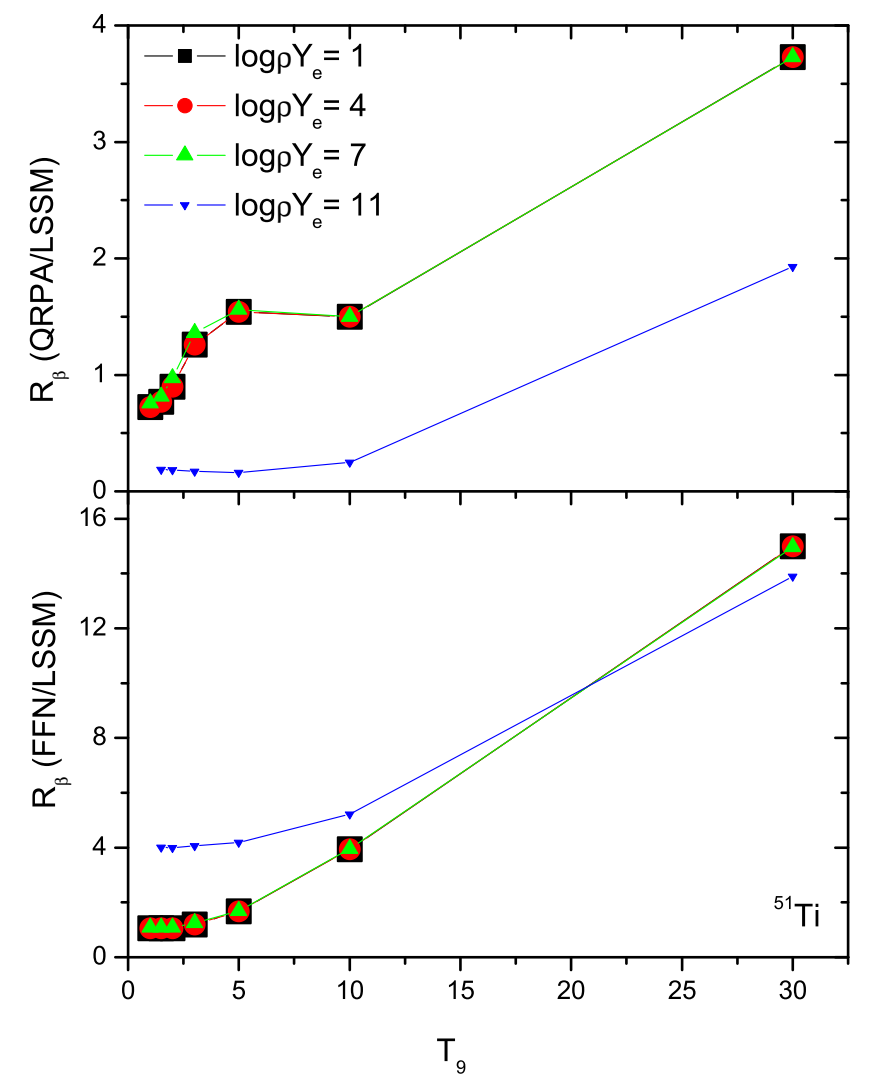

Fig. 1. (Color online) Ratio of reported $\beta$-decay rates to those calculated using LSSM (upper panel) for ${ }^{51} \mathrm{Ti}$ as function of stellar temperatures and densities. T9 gives the stellar temperature in units of $10^{9} \mathrm{~K}$. In the legend, $\log \rho Y_{e}$ gives the $\log$ to base 10 of stellar density in units of $\mathrm{gcm}^{-3}$. The lower panel shows the corresponding ratio of FFN $\beta$-decay rates to those calculated using LSSM.

tial and final states matter at low temperatures and densities, it is the total $\mathrm{B}(\mathrm{GT})$ strength that matters at high temperatures and densities. Brink's hypothesis is not assumed in this calculation (which was adopted in LSSM calculation of weak rates). At high temperatures, $\mathrm{T}_{9}[K]=30$, the agreement is very good hinting towards the fact that the total $\mathrm{B}(\mathrm{GT})$ strength calculated in both models match reasonably well. The lower panel shows that FFN rates are again in reasonable comparison with LSSM rates except at high temperatures for reasons mentioned above.

The agreement with LSSM $\beta$-decay rates for the odd-A nucleus, ${ }^{53} \mathrm{Ti}$, is reason- 


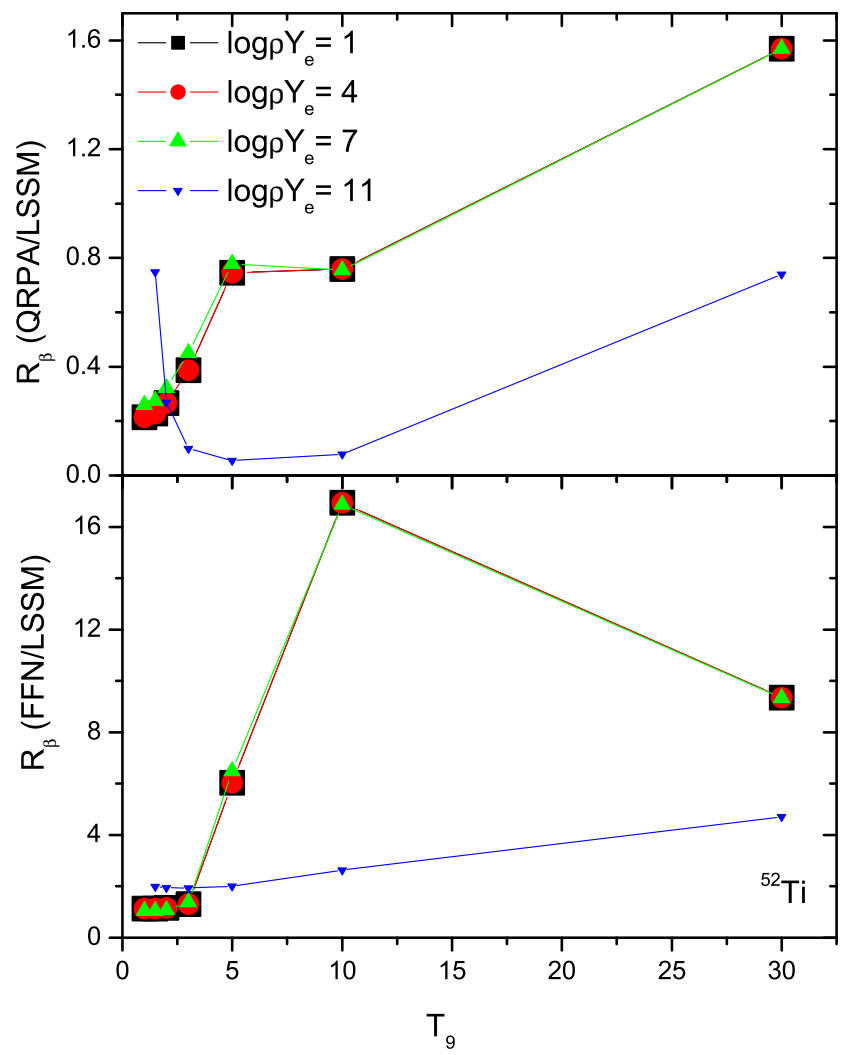

Fig. 2. (Color online) Same as Fig.1. but for $\beta$-decay of ${ }^{52} \mathrm{Ti}$

able (within a factor 5) and depicted in Figure 3. However the pn-QRPA rates are bigger by a factor of thirteen at high temperatures. Comparison between FFN and LSSM calculations (lower panel) reveals that FFN $\beta$-decay rates are much bigger specially at high temperatures. Authors in Ref. 9 reported that, for even-even and odd-A nuclei, FFN systematically placed the back resonance at much lower energies and concluded that contribution of the back resonances to the $\beta$-decay rates for these nuclei decreases. They estimated that LSSM $\beta$-decay rates as a result were smaller, on the average, by a factor of 20 (40) as compared to the FFN $\beta$-decay rates for even-even (odd-A) nuclei.

FFN did not calculate the $\beta$-decay rates of ${ }^{54} \mathrm{Ti}$ and as such a mutual comparison of the three calculations was not possible for this even-even isotope of titanium. We note from Figure 4 that our $\beta$-decay rates of ${ }^{55} \mathrm{Ti}$ are slightly bigger (factor of 


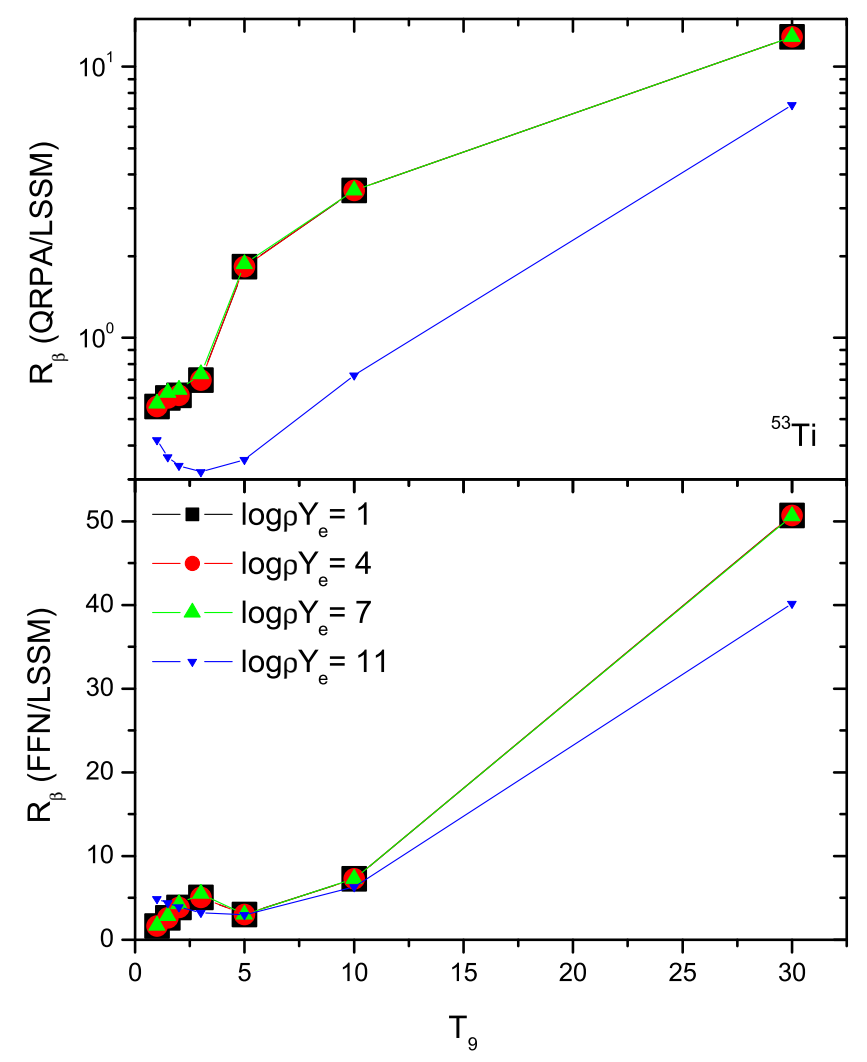

Fig. 3. (Color online) Same as Fig.1. but for $\beta$-decay of ${ }^{53} \mathrm{Ti}$

2 - 3) than the LSSM rates at $\rho Y_{e}\left[\mathrm{gcm}^{-3}\right]=10^{1}, 10^{4}, 10^{7}$. At high densities the LSSM rates exceed the pn-QRPA rates as in previous cases. At high stellar temperatures, the reported rates are more than an order of magnitude bigger than LSSM $\beta$-decay rates. At higher temperatures excited state GT strength distributions are required for the calculation of weak rates (parent excited states have a finite probability of occupation). The LSSM employed the Brink's hypothesis in the electron capture direction and back-resonances in the $\beta$-decay direction to approximate the contributions from high-lying excited state GT strength distributions. On the other hand the pn-QRPA model performs a microscopic calculation of the GT strength distributions for all parent excited states and provides a fairly reliable estimate of the total stellar rates. The lower panel of Figure 4 shows a whopping seven orders of magnitude bigger FFN decay rates at low temperatures and high densities. How- 


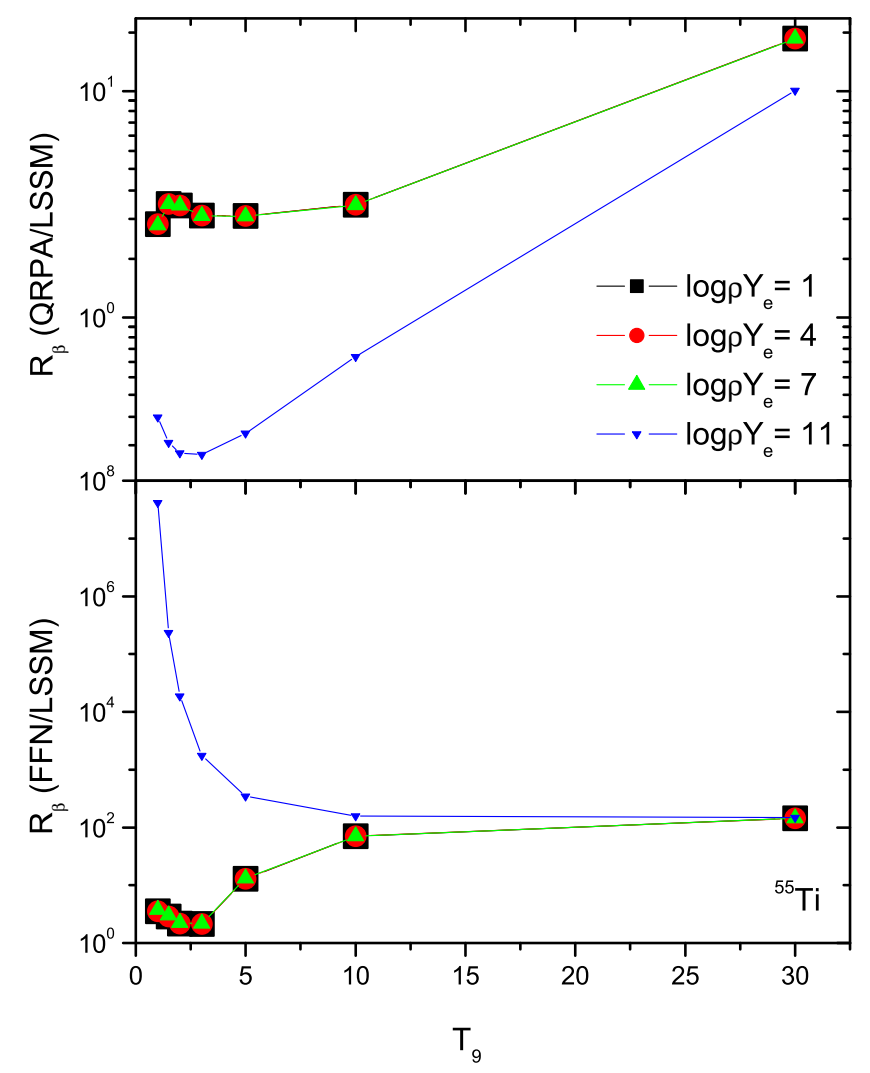

Fig. 4. (Color online) Same as Fig.1. but for $\beta$-decay of ${ }^{55} \mathrm{Ti}$

ever for the same temperature and density domain the two microscopic calculations (pn-QRPA and LSSM) are in good agreement hinting towards the fact that FFN overestimated their $\beta$-decay rates by orders of magnitude. The centroids of the GT distribution functions determine the effective energy of the capture and decay reactions. FFN estimated the GT contributions to the rates by a parametrization based on the independent particle model and estimated the GT centroids using zerothorder $(0 \hbar \omega)$ shell model. Stellar decay rates are fragile functions of the available phase space, $\left(Q_{\beta}+E_{i}-E_{j}\right)$. It is worth mentioning that these $\beta$-decay rates can change by orders of magnitude by a mere change of $0.5 \mathrm{MeV}$, or less in available phase space and are more reflective of the uncertainties in the calculation of energies. It is to be noted that whereas pn-QRPA and LSSM used the value of 7.34 $\mathrm{MeV}$ and $7.81 \mathrm{MeV}$, respectively, as Q-value for this decay reaction, FFN used a 
much larger value of $8.64 \mathrm{MeV}$ for the same reaction.

The comparison of LSSM and pn-QRPA $\beta$-decay rates of ${ }^{56} \mathrm{Ti}$ is similar to the previous case of ${ }^{55} \mathrm{Ti}$ (see Figure [5). At $\mathrm{T}_{9}[K]=30$ the reported decay rates are

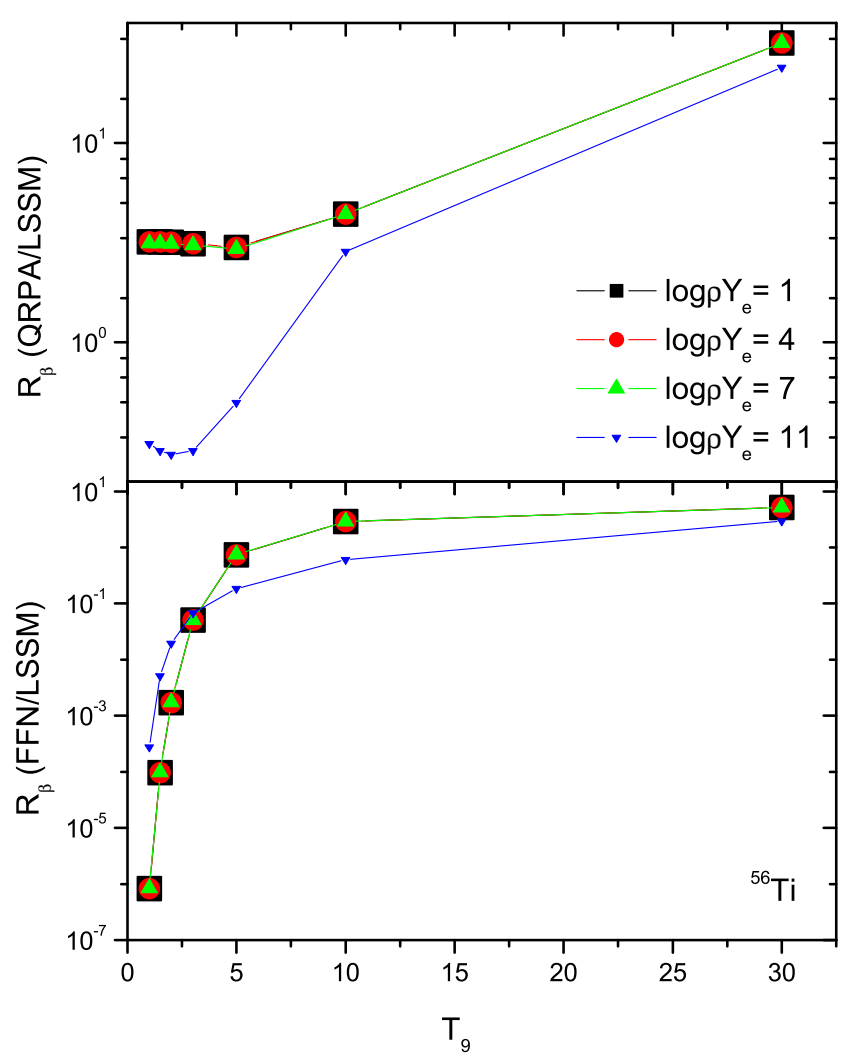

Fig. 5. (Color online) Same as Fig.1. but for $\beta$-decay of ${ }^{56} \mathrm{Ti}$

around a factor of thirty bigger than LSSM rates. For a change we notice in the lower panel that the FFN decay rates of ${ }^{56} \mathrm{Ti}$ are much smaller than the LSSM rates. The rates are more than six orders of magnitude smaller at low temperatures and densities. For the same physical conditions the reported rates are again in far better agreement with the LSSM numbers again hinting toward some problems in the FFN calculations. It was found by authors in Ref. 2 that the approach of FFN was not always reliable in its estimates of the location of GT strength. Unmeasured matrix elements for allowed transitions were assigned an average value of $\log f t=5$ 
in FFN calculations. Measurements were performed for the $\beta$-decay of ${ }^{56} \mathrm{Ti} 21$ after the FFN calculations. This measurement was properly incorporated in the microscopic calculation of pn-QRPA (this work) and LSSM and resulted in a much bigger $\beta$-decay of ${ }^{56} \mathrm{Ti}$.

\section{Conclusions}

Titanium isotopes are amongst the key iron-regime nuclei that play a significant role in the late phases of stellar evolution of massive stars. The $\beta$-decay rates of these isotopes increase the value of the lepton-to-baryon fraction $\left(Y_{e}\right)$ during the late phases of stellar evolution. The temporal variation of $Y_{e}$ within the core of a massive star has a pivotal role to play in the stellar evolution and a fine-tuning of this parameter at various stages of presupernova evolution is the key to generate an explosion. Six isotopes of titanium, ${ }^{51,52,53,54,55,56} \mathrm{Ti}$, were short-listed as important $\beta$-decay nuclei as the product of their abundance and $\beta$-decay rates can cause a substantial change in the time rate of change of lepton-to-baryon fraction. The pnQRPA model was used to calculate the $\beta$-decay rates of these six titanium isotopes in a microscopic fashion. The pn-QRPA model has two distinct and important advantages as compared to other models. Firstly it can handle any arbitrarily heavy system of nucleons since the calculation is performed in a luxurious model space of up to 7 major oscillator shells. Further it is the only available model that can calculate all excited state GT strength distributions in a microscopic fashion which greatly increases its utility in stellar calculations.

The $\beta$-decay rates were calculated on a detailed density-temperature grid point. The positron capture rates on titanium isotopes were also calculated and it was shown that during the presupernova evolution of massive stars the positron capture rates can be safely neglected in comparison with the $\beta$-decay rates. The ASCII files of the calculated rates can be requested from the corresponding author. The pn-QRPA $\beta$-decay rates were also compared against previous calculations. Our study validates the finding of authors of Ref. 9 that FFN systematically placed the back resonance at much lower energies. Consequently the FFN overestimated the $\beta$-decay rates for even-even and odd-A nuclei. The comparison with LSSM calculation is generally fair. However at high densities the LSSM rates are enhanced whereas at high temperatures the pn-QRPA $\beta$-decay rates are much bigger. The study also stresses on the fact that the Brink's hypothesis and back resonances (employed in previous calculations) are not a good approximation and it is desirable to microscopically calculate all excited state GT strength distribution functions for a reliable estimate of stellar $\beta$-decay rates. Core-collapse simulators might find it useful to employ the pn-QRPA $\beta$-decay rates as an alternate (microscopic and reliable) nuclear physics input in their codes. 


\section{Acknowledgements}

The authors wish to acknowledge the support of research grant provided by the Higher Education Commission through the HEC Project No. 20-1171. JUN also wishes to acknowledge the support of research grant provided by the Higher Education Commission Pakistan, through the HEC Project No. 20-1283.

\section{References}

1. H. A. Bethe, G. E. Brown, J. Applegate, J. M. Lattimer, Nucl. Phys. A324, (1979) 487.

2. M. B. Aufderheide, I. Fushiki, S. E. Woosley, D. H. Hartman, Astrophys. J. Suppl. 91, (1994) 389.

3. R. Buras, M. Rampp, H. T. Janka, K. Kifonidis, Phys. Rev. Lett. 90, (2003) 241101.

4. R. Buras, H. T. Janka, M. Rampp and K. Kifonidis K, Astron. Astrophys. 457 (2006) 281.

5. A. Burrows, E. Livne, L. Dessart and C. D. Ott, Astrophys. J. 645 (2006) 534.

6. S. E. Woosley and A. Heger, Phys. Rep.442 (2007) 269.

7. G. M. Fuller, W. A. Fowler and M. J. Newman, Astrophys. J. Suppl. Ser. 42 (1980) 447; 48 (1982) 279; Astrophys. J. 252 (1982) 715; 293 (1985) 1.

8. C. D. Goodman, C. A. Goulding, M. B. Greenfield, J. Rapaport, D. E. Bainum, C. C. Foster, W. G. Love, F. Petrovich, Phys. Rev. Lett 44, (1980) 1755.

9. K. Langanke and G. Martínez-Pinedo, Nucl. Phys. A 673 (2000) 481.

10. J.-U. Nabi, H. V. Klapdor-Kliengrothaus, Atomic Data and Nuclear Data Tables 88, (2004) 237.

11. J.-U. Nabi, H. V. Klapdor-Kleingrothaus, Eur. Phys. J. A5, (1999) 337.

12. J.-U. Nabi, M.-U. Rahman, Phys. Lett. B 612, (2005) 190.

13. J.-U. Nabi, M. Sajjad, Phys. Rev. C 76, (2007) 055803.

14. J.-U. Nabi, M. Sajjad, Phys. Rev. C 77, (2007) 055802.

15. J.-U. Nabi, Eur. Phys. J. A40, (2009) 223.

16. J.-U. Nabi, M. Sajjad, M.-U. Rahman, Acta. Phys. Polon. B 38, (2007) 3203.

17. J.-U. Nabi, Int. J. Mod. Phys. E 19, (2010) 1.

18. G. P. Yost et al. (Particle Data Group), Phys. Lett. B 204, (1988) 1.

19. N. B. Gove, M. J.Martin, Nuclear Data Tables 10, (1971) 205.

20. J.-U. Nabi, H. V. Klapdor-Kliengrothaus, Atomic Data and Nuclear Data Tables 71, (1999) 149.

21. T. Dörfler et al. Phys. Rev. C 54, (1996) 2894. 